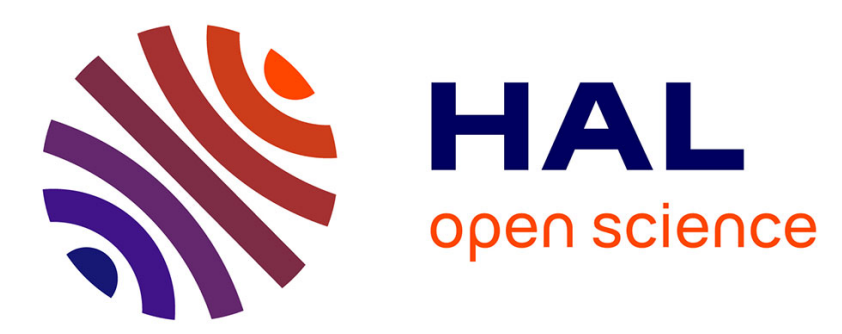

\title{
L'évolution du concept de reconversion : de la substitution d'activité au redéveloppement des territoires
}

Sylvie Daviet

\section{- To cite this version:}

Sylvie Daviet. L'évolution du concept de reconversion : de la substitution d'activité au redéveloppement des territoires. Territoires européens du charbon, Presses universitaires de Provence, pp.243-255, 2006, 10.4000/books.pup.6288 . hal-03171460

\author{
HAL Id: hal-03171460 \\ https://hal.science/hal-03171460
}

Submitted on 19 Mar 2021

HAL is a multi-disciplinary open access archive for the deposit and dissemination of scientific research documents, whether they are published or not. The documents may come from teaching and research institutions in France or abroad, or from public or private research centers.
L'archive ouverte pluridisciplinaire HAL, est destinée au dépôt et à la diffusion de documents scientifiques de niveau recherche, publiés ou non, émanant des établissements d'enseignement et de recherche français ou étrangers, des laboratoires publics ou privés. 


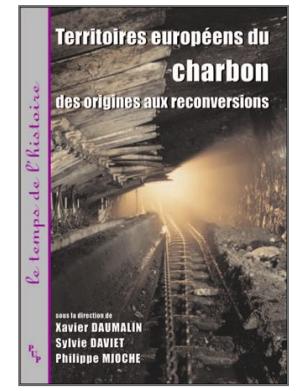

Xavier Daumalin, Sylvie Daviet et Philippe Mioche (dir.)

Territoires européens du charbon

Des origines aux reconversions

Presses universitaires de Provence

\section{L'évolution du concept de reconversion : de la substitution d'activité au redéveloppement des territoires}

\section{Sylvie Daviet}

DOI : 10.4000/books.pup. 6288

Éditeur : Presses universitaires de Provence

Lieu d'édition : Presses universitaires de Provence

Année d'édition : 2006

Date de mise en ligne : 7 juillet 2017

Collection : Le temps de l'histoire

ISBN électronique : 9782821882799

\section{Sobooks}

http://books.openedition.org

Ce document vous est offert par Aix-Marseille Université (AMU)

\section{Aix Marseille universite}

Référence électronique

DAVIET, Sylvie. L'évolution du concept de reconversion : de la substitution d'activité au redéveloppement des territoires In : Territoires européens du charbon : Des origines aux reconversions [en ligne]. Aix-enProvence : Presses universitaires de Provence, 2006 (généré le 16 mars 2021). Disponible sur Internet : <http://books.openedition.org/pup/6288>. ISBN : 9782821882799. DOI : https://doi.org/ 10.4000/books.pup.6288. 


\title{
L'évolution du concept de reconversion : de la substitution d'activité au redéveloppement des territoires
}

Sylvie DAVIET

\begin{abstract}
^̀ Gardanne comme en Europe, l'exploitation du charbon a occupé une Aplace essentielle dans la construction des territoires, façonnant durablement leur histoire économique et sociale, leurs paysages et leur culture. Des Asturies à la Silésie, des Galles du Sud à la Wallonie, de la Lorraine à la Ruhr, du Nord-Pas-de-Calais à la Provence, de nouvelles conurbations sont nées avec la révolution industrielle (Holz, 1977), caractéristiques du continent européen et à l'origine de la construction européenne (Leboutte, 1997). Engagés, pour la plupart, dans un processus de déclin ou de fermeture, ces « pays noirs » posent des problèmes de reconversion et de réaménagement de leurs espaces (Berkner, 1999), affectant la vie de nombreuses communautés minières (Beyon, Hudson, Sadler, 1991). Certaines de leurs installations figurent désormais comme éléments de patrimoine, symboles de temps révolus. C'est plus précisément avec le déclin des charbonnages qu'a pris forme le concept de reconversion dès les années $1950^{1}$; un concept qui, par la suite, s'est étendu à l'ensemble des vieilles régions mono-industrielles en difficulté. Il ne s'agit pas ici de refaire l'histoire des actions engagées, mais de dégager les apports spécifiques de cette expérience pour une compréhension plus générale des processus de mutation des activités. Partant de la situation française et de la récente fermeture de ses deux derniers bassins, celui de Provence en janvier 2003 et celui de Lorraine en avril 2004, nous examinerons l'évolution de ce concept et les difficultés rencontrées dans sa mise en œuvre pour faire des anciennes régions minières de nouveaux territoires porteurs d'avenir.
\end{abstract}

1 Mais dès l'entre-deux-guerres, nombre de bassins anglais sont déjà entrés en crise. 
Le terme de reconversion a désigné, dans un premier temps, les dispositifs mis en place par les pouvoirs publics pour amortir les effets de la baisse des effectifs ou de la fermeture d'établissements, en favorisant le développement de nouvelles activités. En vérité, dans les sites industriels confrontés au déclin d'une activité jusqu'alors motrice et dominante, on s'est vite aperçu qu'en touchant à l'activité c'est tout un système qui s'effondrait. De ce fait, les politiques de reconversion se sont profondément transformées depuis une quarantaine d'années, en passant de politiques centrées sur la substitution d'activité à des politiques plus globales de redéveloppement des territoires. On peut dire à présent que le phénomène de reconversion renvoie à la remise en cause d'un système économique, social, culturel et spatial sous la poussée d'un nouveau système comprenant des activités et des espaces concurrents (Daviet, 1990). Il désigne un processus de réorganisation qui affecte les hommes, les entreprises et l'espace, et qui est le plus souvent synonyme de crise : crise de l'emploi, crise démographique parfois, crise de confiance et d'identité. L'idée que les problèmes de conversion font système a contribué, dans les régions minières plus qu'ailleurs peut-être, à l'élaboration du concept de territoire comme ensemble d'interactions entre les hommes, les activités et l'espace. Parler de la reconversion recouvre donc des dimensions multiples que je regrouperai en trois grands volets, distincts pour des raisons formelles mais en réalité étroitement liés entre eux : la diversification des activités et les nouvelles formes de développement; la reconversion institutionnelle et la recomposition des territoires ; le travail du deuil et la construction d'une identité nouvelle.

\section{La diversification des activités et les nouvelles formes de développement}

Jacques Chérèque qui fut dans les années 1980 un acteur majeur de la reconversion en Lorraine rappelait récemment ${ }^{2}$ à propos de la sidérurgie : « Il fallait montrer qu'il y avait une vie après la sidérurgie ». Par ces termes, il soulignait que la dimension de l'espace vécu est essentielle. Nombre de régions minières et sidérurgiques sont, en effet, passées d'un modèle de croissance à un modèle de crise, donnant l'impression que l'on assistait à une inversion de la théorie des pôles de croissance (Rinaldi, 1986). Les territoires à reconvertir sont apparus à l'extrême comme des pôles de décroissance difficilement réversibles, caractérisés par la montée du chômage, la fermeture des commerces et

2 Aux journées géographiques de Nancy consacrées au thème de la reconversion (2930 septembre 2004). 
services, l'émigration des jeunes actifs, la baisse des prix de l'immobilier et des terrains qui creuse le cercle vicieux de l'appauvrissement des populations, les friches industrielles et urbaines, le sentiment de désespoir... Face à la menace d'une mort imminente, il s'est agi, pour les acteurs locaux et nationaux, de redonner la vie, en permettant à la population de se maintenir sur place et d'y trouver du travail.

Dans les bassins houillers français, la situation est spécifique. Le statut du mineur a presque toujours permis d'éviter les licenciements et les dispositifs de reconversion, au sens large, se sont échelonnés sur de longues périodes antérieures aux fermetures ${ }^{3}$, empêchant le plus souvent les situations extrêmes que je viens de citer. La baisse des effectifs miniers a été obtenue par trois grands types de mesures : d'une part des mesures sociales, comme les départs en préretraites, avec en particulier le CCFC (Congé charbonnier de fin de carrière) ${ }^{4}$; d'autre part des mesures de conversion du personnel, comme en direction d'Électricité de France, pouvant entraîner le cas échéant une mobilité extrarégionale ; enfin des mesures de diversification des activités pouvant, selon les cas, bénéficier ou non à la population minière, en fonction du profil des emplois concernés.

La diversification des activités a connu des temporalités et des rythmes différents, selon les bassins et selon les périodes. Selon les bassins, car, en fonction de leur compétitivité, les fermetures se sont échelonnées des années 1960 à nos jours. Selon les périodes car, en fonction du marché de l'énergie, la politique charbonnière a évolué. La grande récession des années 1960 constitue une première phase fondatrice. Des zones d'activité sont mises en réserve. La conjoncture économique est encore favorable. La SOFIREM (Société financière pour la reconversion des régions minières) est créée en 1967 pour :

«prendre des participations minoritaires dans des entreprises devant faciliter l'embauche des mineurs appelés à quitter leur emploi. »

Avec la crise pétrolière des années 1970 s'ouvre une seconde phase, moins propice à la diversification pour deux raisons. D'une part, la stabilisation, voire la relance de la production charbonnière, entraîne globalement une mise en sommeil des dispositifs de conversion. D'autre part, le contexte économique global est devenu défavorable à l'emploi

3 Cf. le dossier de presse de Philippe de La Doucette, PDG de Charbonnages de France, mars 2004.

4 Ces mesures évitent un effondrement de l'activité en maintenant le pouvoir d'achat mais ont des conséquences sociales néfastes chez des actifs encore jeunes qui se retrouvent désœuvrés (cf. la communication de J. Domenichino et J.-M. Guillon). 
dans un nombre croissant de secteurs. Néanmoins, les implantations qui s'effectuent au cours de cette période portent en germe un nouveau tissu non négligeable d'activités. Depuis la fin de la relance charbonnière, s'ouvre une troisième phase de réactivation et d'intensification de la reconversion qui se déroule en deux temps. Tout d'abord, 1984 marque l'arrêt définitif des embauches dans l'ensemble des Charbonnages de France. Des fonds d'industrialisation sont créés, tandis que les opérations internes de reclassement du personnel s'accélèrent vers les sièges les plus compétitifs de Lorraine et de Provence. Depuis 1994, la perspective de fermeture définitive des derniers sièges est annoncée. La signature du Pacte Charbonnier vient alors accélérer la démotivation du personnel et le flux des départs. Les actions de diversification disposent de moyens accrus avec les fonds structurels européens, mais prennent place dans un marché européen où la concurrence des territoires s'est exacerbée et dans une économie davantage mondialisée.

Au cours de ces quatre décennies, la diversification des activités a donc progressé tout en rencontrant deux grands types de difficultés. Premièrement, elle a été marquée par des modèles de développement qui sont devenus obsolètes et ont dû évoluer au fur et à mesure que la structure de l'économie globale changeait et que la société était plus sensible à l'environnement et au cadre de vie, incluant le développement du tourisme et des loisirs. Deuxièmement, elle s'est trouvée au départ limitée par l'héritage structurel d'une industrie de main-d'œuvre faiblement qualifiée et d'un système de formation devenu inadapté. La nécessité est vite apparue d'œuvrer non seulement pour la reconversion des mineurs eux-mêmes mais aussi, et de plus en plus, pour les enfants de mineurs, en prenant en compte le facteur temps et l'évolution des systèmes de formation à l'échelle au moins d'une génération.

Dans ce contexte, la priorité a longtemps été donnée à l'industrie, en tant que moteur du développement, avant que la recomposition des systèmes productifs n'incline à accorder plus de place aux services aux entreprises. Cette priorité est aussi à mettre en lien avec l'identité des acteurs de la reconversion. Les groupes industriels, en particulier CDF, ont été associés au processus de conversion et en sont devenus les « chevilles ouvrières ». La SOFIREM a servi de modèle à d'autres sociétés du même type (notamment dans la sidérurgie), tandis que les services Industrialisation prospectaient et assuraient le montage des dossiers d'implantation. On peut souligner à cet égard l'importance accordée par les acteurs au développement exogène et aux réalisations ayant une certaine visibilité. Les facteurs mis en avant pour attirer les investissements extérieurs ont eux-mêmes évolué. À la logique des 3 P (Place, Personnel, Prime), d'autres paramètres se sont surimposés comme les perspectives de développement du marché, les services partagés, la gestion des 
déchets... L'environnement des entreprises en termes de formation, recherche, infrastructures de transport... a nourri une approche de plus en plus étoffée des conditions de l'activité et du développement. Des facteurs de localisation à l'ancrage territorial des entreprises, on a observé un changement de paradigme motivé par un contexte plus général de redéploiement des activités (à l'échelle européenne et mondiale), de mobilité des capitaux internationaux et de dumping social. Si au cours des années 1980, les discours et les efforts ont davantage porté sur le développement endogène, en mettant en avant la création d'entreprise et les PME-PMI, les stratégies et les dispositifs traduisent à l'heure actuelle une plus grande mixité des approches, combinant l'attraction de grands projets emblématiques et la construction sur place d'un maillage d'entreprises. En définitive, les politiques de reconversion des régions minières ont peu à peu perdu de leur spécificité pour des raisons internes et externes : en interne, leur caractère mono-industriel s'est atténué ; en externe, elles ont été confrontées de façon croissante aux mêmes logiques globales que les autres régions. Elles se sont donc rapprochées des stratégies communes aux autres territoires.

Mais au-delà de ces tendances communes, les résultats ont été différents selon les régions, en fonction de quatre grands critères essentiels qui tiennent à la taille des bassins, à leur situation géographique, au poids relatif de la mine dans l'économie locale d'origine, à la nature des nouvelles activités en situation de filiation ou de rupture avec les métiers de la mine. À titre d'exemple, les différences sont évidentes entre : un bassin houiller lorrain qui compte 25000 mineurs en 1984 et le bassin provençal qui en compte 2000 à la même date ; une région du NE français qui connaît un solde migratoire déficitaire depuis les années 1970 et une région provençale qui bénéficie dans le même temps d'un effet sun-belt au point que l'on parle de «France inverse » (Uhrich, 1987) ; entre un bassin frontalier distant de sa métropole régionale $(\mathrm{Metz})^{5}$, et un autre situé au cœur d'une aire métropolitaine (Marseille) de plus d'un million d'habitants.

De ce fait, la Lorraine se trouve, dans le contexte actuel, moins attractive que la Provence, en dépit d'atouts comme la frontière et le bilinguisme de sa population est-mosellane. L'importance de la population minière a conduit le Service Industrialisation à prospecter avec des stratégies sectorielles garantissant la durabilité des emplois (éviter les usines tournevis) mais aussi leur compatibilité avec le niveau de qualification de la main-d'œuvre régionale. Le tissu d'entreprises

5 Même si Forbach se trouve dans l'aire d'influence de Sarrebruck, la Sarre n'est pas son territoire de rattachement politique et administratif. 
implantées, souvent d'origine allemande, recouvre des secteurs variés où dominent à présent, comme dans le Nord-Pas-de-Calais, la plasturgie et l'automobile (Delmer-Lantreibecq, 1996). Après Continental, General Motors, Textar et Girling dans les années 70 et 80, l'implantation la plus emblématique des années 1990 est celle des usines de fabrication de la Smart (groupe Daimler-Chrysler) et du Parc Intégré de Fournisseurs sur la zone d'Hambach (2 500 emplois); l'ensemble constitue la forme la plus accomplie du « juste à temps ». $20 \%$ des salariés sont des femmes, ce qui constitue une nouveauté appréciable dans une région où la structure de l'emploi restait jusqu'à présent très masculine. Les créations d'emplois depuis 1984 compenseraient, d'après les données officielles, les quelque 20000 postes supprimés. La reconversion a bénéficié principalement au renouveau d'une fonction industrielle plus diversifiée, mais les services sont encore peu développés et le niveau de qualification de la main-d'œuvre insuffisant. Le plan Chérèque de 1988 qui visait à faire de la Moselle-Est un pôle d'excellence des échanges franco-allemands n'a pas abouti à des réalisations concrètes, si ce n'est le développement d'une activité logistique peu pourvoyeuse d'emplois.

Situé entre Aix et Marseille et bénéficiant d'une excellente desserte autoroutière, le bassin de Provence est en proie depuis les années 1970 à une péri-urbanisation intense (Ferreira, 1988). La microélectronique est le principal secteur qui s'y est développé depuis 1979, en particulier sur la commune de Rousset où les deux donneurs d'ordres STMicroélectronics et Atmel sont localisés (Daviet, 2002). La microélectronique représente 80 \% des emplois aidés depuis 1994. D'origine exogène, cette industrie de haute technologie a trouvé in situ sa dynamique propre de développement, débordant largement les limites du bassin. Les 6000 emplois créés depuis 10 ans dans le périmètre aidé compensent nettement en volume les 2000 emplois miniers disparus. Toutefois, les nouvelles entreprises sont régulièrement menacées de délocalisation, en dépit d'importants investissements. D'autre part, cette nouvelle activité correspond à des logiques techniques et sociales en rupture complète avec le monde de la mine (Rychen, Zimmermann, 2000) et a, le plus souvent, bénéficié à des populations très qualifiées non autochtones. La reconversion des mineurs eux-mêmes, au demeurant peu nombreux, n'a pas constitué un objectif prioritaire en Provence, contrairement à ce qui s'est produit en Lorraine. Statistiquement, la reconversion de mineurs actifs dans le bassin de Gardanne n'a concerné qu'une centaine de personnes (Chelu, Daviet, 2003). La périurbanisation et l'industrialisation de l'espace se sont combinées pour générer une pression foncière et immobilière qui est source de tensions.

Au-delà de ces spécificités, les nouvelles implantations se sont souvent établies, en Provence comme en Lorraine, à la périphérie des 
bassins, introduisant des disparités importantes avec le cœur des zones minières où se concentrent les héritages paysagers et sociaux d'une activité devenue moribonde. Ces disparités génèrent des recompositions territoriales et institutionnelles qui nous amènent à sortir du champ de l'économie pour aborder celui de la géopolitique locale. Nous allons voir de ce fait comment la crise industrielle et le processus de reconversion ont modifié le système local d'action publique.

\section{Reconversion institutionnelle et recompositions territoriales}

La différentiation interne des bassins entre la périphérie et le centre conduit à définir la notion de «bassin », en posant la question du périmètre des zones à aider et de leur évolution. Au temps fort de l'activité minière, l'aire de recrutement des mineurs s'est étendue sur les communes rurales périphériques, constituant un bassin d'emploi qui dépassait largement la zone des emprises minières où se concentrait le peuplement. Autour de Gardanne, c'est un ensemble de 17 communes qui sont concernées ${ }^{6}$. Dans le bassin lorrain, la zone minière occupait une part significative de l'arrondissement de Forbach; le bassin de recrutement s'étendait aux arrondissements voisins de Boulay à l'Ouest et de Sarreguemines à l'Est, sur un ensemble d'environ 250 communes $^{7}$. Au début du processus de reconversion, les périmètres aidés ont été légitimement définis à l'ensemble des « bassins » d'emploi miniers. C'est donc dans les périphéries offrant des espaces disponibles et une bonne accessibilité que les premières zones d'activités ont été logiquement créées, tandis que l'exploitation se poursuivait au centre. Le succès de ces zones a modifié en profondeur la géographie de l'emploi et de la production de richesse au sein des bassins. À l'heure de la fermeture, l'ancienne définition des «bassins » reste dans les mémoires mais a perdu fonctionnellement sa pertinence originelle. Les communes-centre apparaissent comme les parents pauvres de la reconversion, perdent le bénéfice de la redevance des mines et concentrent le coût de la réhabilitation des paysages. La prise en compte de cette évolution, pourrait conduire à recentrer une part plus importante des aides sur les communes-centre, si l'on considère que le principe des zonages est d'accorder un avantage comparatif aux territoires en difficulté. À titre d'exemple, l'arrondissement de Sarreguemines, qui a notamment bénéficié de l'Europôle de la Smart, ne perçoit plus aujourd'hui la prime d'aménagement du territoire.

6 Soit environ 100000 habitants.

7 Soit environ 350000 habitants. 
Force est de constater l'enjeu politique de ces zonages. En évoquant les rivalités inter-communales qui se sont manifestées, en Lorraine comme ailleurs, Jacques Chérèque rappelait que : « les collectivités locales n'ont pas toujours joué le jeu $»^{8}$, c'est-à-dire le jeu d'une reconversion concertée et équitable. En disant cela, il posait avec acuité la question du jeu des acteurs et de la concurrence des territoires à l'intérieur des bassins ; le jeu des acteurs faisant ainsi partie intégrante du processus de reconversion lui-même. L'absence d'intercommunalité a souvent constitué un handicap, à l'heure où les instances locales ont de plus en plus été incitées à participer à l'évolution du tissu économique. Sur le plan institutionnel aussi, on peut donc dire que les régions minières connaissent une sorte de normalisation, en s'inscrivant dans un mouvement plus général de recomposition des territoires, c'est-à-dire d'ajustement entre les cadres territoriaux d'exercice du pouvoir et les espaces de vie cohérents en matière d'aménagement et de développement.

L'émergence du pouvoir communal revêt toutefois une spécificité dans les bassins miniers car le pouvoir industriel s'est longtemps substitué au pouvoir communal ou urbain traditionnel. La mine était maîtresse du sol, organisatrice des logements et des équipements..., de la vie associative et culturelle..., de la vie politique et syndicale à travers l'appartenance des notabilités, comme d'une large part de la population, à la même communauté professionnelle. Elle a modelé les infrastructures matérielles et les superstructures mentales, tout en constituant un univers traversé de contradictions internes et de conflits sociaux plus ou moins violents. L'internalisation du pouvoir d'aménagement par la grande entreprise a en quelque sorte suppléé la fonction citadine. La dissolution de l'entreprise aujourd'hui se traduit par une externalisation de ses fonctions sociales en direction d'acteurs variés. Ces transferts caractérisent la situation de reconversion comme un système complexe de mutations non seulement économiques et sociales mais aussi politiques et institutionnelles. Les Services Industrialisation qui pilotaient la reconversion à l'échelle du bassin ont eu du mal, par exemple, à se muer en agence de développement à vocation intercommunale. Le désengagement de l'acteur leader, qui représentait conjointement l'entreprise et les pouvoirs publics, fait parfois perdre en cohérence. Il n'y a plus d'entité stable gérant une crise, mais plutôt une construction mouvante affectée par la situation de crise (Grossetti et alii, 1998).

8 Table ronde des journées géographiques de Nancy consacrées au thème de la reconversion (29-30 septembre 2004). 
Dans le bassin lorrain, une association est créée en 1989, à l'initiative de la sous-préfecture et des HBL qui anticipent la transition vers de nouvelles instances. «Actipromo » regroupe alors, avec ses membres fondateurs, des élus, des syndicalistes et divers partenaires de la vie locale. Elle coordonne plusieurs opérations touchant au développement comme à la promotion du bassin rebaptisé « Moselle-Est». Cependant Actipromo n'emporte pas l'adhésion de tous et certaines communes n'y adhèrent pas. L'évolution vers une nouvelle entité institutionnelle peine à émerger, traduisant la permanence de rivalités urbaines et d'antagonismes entre un bassin urbain et une périphérie rurale. L'association devient moribonde. Cependant, en 2003, l'AGEME (Agence pour l'Expansion de la Moselle-Est) se constitue, regroupant l'essentiel des communautés de communes de l'ensemble du « bassin » dans son périmètre initial, soit 12 structures intercommunales de Bitche à Bouzonville, en passant par Sarreguemines, Forbach, Freyming-Merlebach, Saint-Avold et Faulquement. Cette agence a pour vocation de :

«prendre le relais de la Mission Industrialisation des Houillères du Bassin de Lorraine en amplifiant ses missions. Surtout il s'agit d'élargir son assise en passant de ce qui était la prise en charge par une seule entreprise - fût-elle publique - à la responsabilité par l'ensemble des acteurs du territoire : collectivités locales et territoriales, entreprises, organismes de formation et de développement, financiers... ${ }^{9}$

En Provence, on n'observe pas de structure équivalente qui réunisse les 17 communes de l'ancien bassin minier. Ce dernier n'est pas parvenu à faire perdurer les anciennes solidarités face à l'ampleur des mutations qui se sont déroulées. Le périmètre des intercommunalités a beaucoup évolué au cours de ces dernières années. Neuf communes ont rejoint la Communauté d'Agglomération du Pays d'Aix (CAPA) ; sept autres forment la communauté de communes « Lou Païs de l'Estello es dou Merlancoun ${ }^{10}$,

9 Selon les termes de l'assemblée générale du 10/12/2003

10 «La communauté de communes "Lou Païs de l'Estello es dou Merlancoun" est ce que l'on appelle un ratage. Trop petite, prise entre les deux monstres que sont "Marseille Provence Métropole" et "Pays d'Aix", elle est transformée en région dortoir pour ses deux grandes voisines. Créée depuis 10 ans, elle n'a ni emblème, ni volonté réelle d'exister. Elle est traversée par deux courants contradictoires : l'un prône le rattachement à "Garlaban Huveaune Ste Beaume" ce qui permet de récupérer le "village gaulois" de Gardanne, l'autre prône la dissolution et le rattachement à l'un ou l'autre des deux voisins : MPM et CAPA. Un seul point d'accord pour tous les hommes politiques de droite comme de gauche : la disparition de la communauté de commune de l'Étoile et du Merlançon. » http://vexil.prov.free.fr/estello/estello.html 
comprenant d'anciennes communes minières comme Gréasque ; tandis que Gardanne se retrouve isolée. Le Service Industrialisation (CDFIndustrialisation) travaille avec Pays d'Aix Développement (structure de développement de la CAPA) et Provence Promotion à l'échelle du département. Il a récemment initié un Dispositif d'Amorçage Provençal destiné à faciliter l'émergence de nouveaux projets innovants dans le «bassin minier de Provence ». Ce dispositif est mis en œuvre par Pays d'Aix Développement, mais peut concerner des communes du «bassin » qui ne font pas partie de la CAPA.

On peut par conséquent observer une divergence significative entre un bassin lorrain qui a conservé une relative cohésion dans ses limites antérieures, tout en œuvrant pour le renouvellement de son identité, et un bassin provençal profondément disloqué dont la représentation symbolique tend à se réduire à l'îlot politique et social de la commune de Gardanne. Cette divergence conduit à s'interroger sur les atouts et les handicaps des mécanismes identitaires dans la reconversion des territoires.

\section{Le travail du deuil et la construction d'une identité nouvelle}

La situation de reconversion résulte d'une volonté politique et suppose un accord sur les actions à engager et coordonner au sein d'un périmètre qui dépasse le plus souvent les limites communales, d'où l'enjeu d'une logique intercommunale. Elle nécessite également un certain consensus parmi les forces politiques et syndicales et plus généralement entre les acteurs sociaux. La situation de reconversion n'est pas donc une donnée objective mais relève d'un processus de construction sociale. Selon Grossetti (1998, p 207) :

«La qualification de la situation comme reconversion industrielle se construit progressivement au cours d'un processus que l'on peut en général décomposer en trois grandes étapes : incrédulité, deuil, action. »

En vérité, ces étapes ne se succèdent pas forcément dans le temps, mais tendent le plus souvent à se chevaucher, en fonction de la différentiation interne des bassins. En effet, tandis que les actions de conversion ont pris naissance dans la périphérie, la poursuite de l'exploitation au centre a entretenu l'espoir d'une pérennisation de l'activité charbonnière et l'épreuve du deuil n'intervient qu'après la cessation définitive de toute exploitation.

Cette question de deuil est essentielle. Elle représente un moment plus ou moins long et douloureux au cours duquel la fin de la mine est progressivement acceptée. Les exemples de refus de cet état de fait sont nombreux et donnent lieu à des conflits: 
«Alès illustre une logique commune à bien des villes minières : refus de la fermeture des puits, parfois violents, puis acceptation progressive, évolution des diagnostics et apparition de projets impliquant plus ou moins les collectivités locales. » (Grossetti, 1998, p 69).

Le refus de la fermeture y a entraîné, à moment donné, le refus de la reconversion. Dans le bassin lorrain, alors que la fin de l'exploitation n'est pas encore engagée :

«L'annonce du plan de récession (de 1984) a suscité de violentes réactions. Il n'était pas question de renoncer au charbon. Les années 1984-1987 ont été celles du doute et de la confusion. Puis la notion de conversion a peu à peu fait son chemin. Avec l'entrée en application du Plan Chérèque et les résultats encourageants de l'industrialisation, l'année 1988 a marqué (provisoirement) le retour de la paix sociale. » (Daviet, 1990).

L'acceptation de la récession, de la fermeture et de la reconversion est conditionnée par les rapports de force politiques et syndicaux au sein du mouvement ouvrier. Elle est faite de phases cycliques où alternent des moments de crise et des moments d'apaisement jusqu'à l'issue finale. À ce moment, le nécessaire travail de deuil ne s'effectue pas automatiquement mais nécessite un accompagnement de la population dans ce qui disparaît et dans ce qui se construit. Il comporte donc une double dimension : la reconnaissance de ce qui a existé et la construction d'une identité nouvelle.

La reconnaissance de ce qui a existé passe par le respect de l'identité minière. Elle donne tout son sens à l'entretien de la mémoire et la conservation du patrimoine. Nombre de travaux montrent que l'espace comporte des mémoires. Il est héritage d'actions antérieures qui tracent des « sillons » dont les sociétés doivent tenir compte (Bonnemaison, 1999, 2000), car les signes du passé témoignent de notre identité. Nous ne pouvons pas tout conserver, mais nous ne pouvons pas non plus « faire table rase du passé » sans remettre en cause notre identité collective. Et le territoire est un espace support d'une identité collective. Il témoigne d'une appropriation par des groupes qui se donnent une représentation d'eux-mêmes, de leur histoire, de leur singularité (Di Méo, 1998). L'espace vécu est ainsi fondateur d'un rapport sensible et culturel au territoire, autrement dit d'une territorialité spécifique (Daviet, 2005). Il ne s'agit pas seulement de garder des symboles paysagers du passé qui seraient comme une coquille vide, mais aussi d'accomplir un travail de valorisation de ce passé. Car il est plus facile de se reconstruire si ce dont nous héritons a du sens et de la valeur. Les actions scientifiques et culturelles qui contribuent à valoriser le passé participent au travail de deuil et constituent une étape permettant d'envisager l'avenir autrement. Les 
cérémonies qui se sont déroulées en Lorraine le 23 avril 2004 à l'occasion de la fermeture ont eu un grand retentissement auprès des médias et des populations qui y ont massivement participé (37000 visiteurs). Le succès de cette manifestation témoigne d'une attente ; il montre que l'hommage rendu aux mineurs n'est pas un vain mot s'il permet de tourner la page du charbon dans la dignité.

La construction d'une identité nouvelle se construit dans le temps. Elle suppose non seulement l'existence de nouveaux emplois, mais aussi la connaissance par la population de ce qu'ils représentent. Elle requiert une appropriation de ces nouveaux territoires de l'activité. Le Bassin Houiller Lorrain a entrepris de se doter d'une nouvelle image et s'est attribué le nouveau nom de «Moselle-Est». Cette évolution est le résultat d'une démarche volontaire et structurée qui a débuté avec la naissance d'Actipromo en 1989. Ses actions de promotion et de communication ont commencé par l'opération « Entreprise Express ». Une exposition itinérante de produits industriels locaux a pris place dans un train qui a parcouru les principales villes-étapes de Moselle-Est au cours de l'année 1990. Un éventail de 70 entreprises y était représenté. Chaque visiteur recevait à l'entrée un baladeur dont la cassette servait de guide. L'Éducation Nationale a participé à l'opération, permettant à des groupes de lycéens de venir visiter le train qui a reçu au total environ 42000 visiteurs. La conduite adoptée était de redonner confiance à la population qui s'identifiait encore à la mine et ne percevait pas la réalité du nouveau tissu industriel, ni ses perspectives d'avenir.

Dans le bassin de Provence, l'essor de la microélectronique est visible et massif mais n'a pas suscité l'adhésion de tous. Les manifestations de communication autour de ce secteur ont été tardives et les opérations de diffusion de sa culture scientifique et technique seraient utiles. Seule l'inauguration de l'usine 8 pouces de ST Microélectronics en 2000 a connu un retentissement important avec la venue du premier ministre Lionel Jospin, annonçant la création d'une nouvelle école d'ingénieurs à Gardanne. Au mois de juin 2004, la municipalité de Gardanne, qui bénéficie alors du développement de ce nouveau secteur, a présenté à la population le nouveau Centre Microélectronique de Provence qui sera achevé à la rentrée 2006. Dans les communes rurales qui ont été les premières bénéficiaires des nouvelles implantations, la problématique est différente, notamment du fait de la pression immobilière et foncière qui s'exerce sur le sol. Les industriels sont du reste conscients des effets négatifs de cette dynamique :

« L'implantation des familles, la structure de leurs dépenses, l'utilisation accrue des infrastructures de transport, l'économie nouvelle induite localement (nouvelles implantations d'entreprises de services liées à la population nouvelle), les effets de richesse induits sur les 
propriétaires des alentours par l'augmentation du prix des terrains et habitations..., ont été de véritables chocs économiques et sociaux. »"

On assiste ainsi à d'autres formes de refus de l'industrialisation de ces territoires, sur la base de la défense d'une identité rurale, contre une industrie devenue conquérante. Cette situation plaide en faveur d'une vision plus équilibrée des actions d'aménagement et de développement, notamment entre Gardanne et la vallée de l'Arc.

\section{Conclusion}

À travers cette présentation, qui pourtant s'est limitée à un nombre restreint d'exemples, on pourra percevoir la multitude des problématiques territoriales qui viennent se greffer autour du processus de reconversion et qui sont générées par lui. De la substitution d'activité au redéveloppement des territoires, les chemins parcourus sont longs et complexes. Le développement de nouvelles activités ne s'explique qu'en partie par les politiques conduites. Les limites d'une généralisation tiennent à la situation géographique des bassins, à l'échelle régionale, nationale et européenne. Elles tiennent également au substrat institutionnel et aux forces politiques et sociales en présence. En France, l'État et CDF ont joué un rôle majeur. Pour CDF, il s'agit désormais de " passer le relais », quand au rôle de l'État, il s'est affaibli dans le contexte d'une décentralisation qui accorde plus de place aux systèmes locaux d'action publique. De ce fait le redéveloppement des territoires ne s'opère pas selon des schémas préétablis et relève d'un processus en devenir. Il est notamment conditionné par des formes de régulation davantage locales et des cycles d'activité devenus plus courts et incertains.

11 C'est ainsi que s'exprime Laurent Gouzènes, Directeur du Plan et Programmes d'Études à STMicroelectronics. 